\title{
Hot Hydrogen in the Jovian Corona
}

\author{
Emerich, C. \\ Institut d'Astrophysique Spatiale, Université Paris-Sud, bâtiment 121, \\ 91405 Orsay, France
}

Ben Jaffel, L.

Institut d'Astrophysique, 98bis bd Arago, 75014 Paris, France

Clarke, J. T.

Center for Space Physics, Boston University, MA02215, USA

Ballester, G.

Lunar \& Planetary Laboratory, University of Arizona, AZ85721, USA

\begin{abstract}
The asymmetry observed in the H-Ly $\alpha$ profiles measured near the Jovian equatorial limbs by the STIS/HST spectrograph is interpreted as due to $\mathrm{H}$ atoms propagating in the upper Jovian atmosphere with velocities $\sim 80 \mathrm{~km} / \mathrm{s}$ relative to Jupiter's rotation. A qualitative scenario is suggested to correlate these energetic $\mathrm{H}$ atoms to energetic neutral particles recently detected by Cassini at higher altitudes.
\end{abstract}

Owing to the unique capabilities of the STIS/HST spectro-imager, Jovian H-Lya line profiles were obtained on different locations of Jupiter, with unprecedented accuracy. For both observations sets obtained around the 2000 and 2001 oppositions, the Jovian line profiles reveal a distinct asymmetry near the two opposite equatorial limbs: the red wings are in excess relative to the blue ones for the receding limbs, the reverse holding for the approaching limbs. We attributed these additional Ly- $\alpha$ emissions to resonant scattering of the solar Ly- $\alpha$ emission by a high altitude population of fast neutral $H$ located close to the approaching and receding limbs. The measured velocities, ranging between a few tens of $\mathrm{km} / \mathrm{s}$ and $\sim 170 \mathrm{~km} / \mathrm{s}$ relative to Jupiter, reveal a population of neutral $\mathrm{H}$ in super corotation with Jupiter. Such a population of fast neutral atoms is likely to be produced by charge exchange between neutral $\mathrm{H}$ and energetic atmospheric and magnetospheric charged particles which are accelerated by the intense Jovian magnetic field. As the large observed velocities evoke energetic neutral atoms (ENA) widely studied for the Earth's atmosphere, we suggest that multiple charge exchange processes should similarly produce secondary ring currents in the lower Jovian atmosphere, where neutral densities are large enough to make charge exchange efficient. Finally, these newly observed energetic atoms (few ev) should be correlated with the more energetic neutral particles (few Kev) recently detected by Cassini at 0.5 UA from Jupiter, through a cascade of charge exchange interactions. 